\title{
Analysis and compensation for the effect of the catheter position on image intensities in intravascular optical coherence tomography
}

Shengnan Liu

Jeroen Eggermont

Ron Wolterbeek

Alexander Broersen

Carol A. G. R. Busk

Helle Precht

Boudewijn P. F. Lelieveldt

Jouke Dijkstra 


\title{
Analysis and compensation for the effect of the catheter position on image intensities in intravascular optical coherence tomography
}

\author{
Shengnan Liu,, Jeroen Eggermont, ${ }^{a}$ Ron Wolterbeek, ${ }^{b}$ Alexander Broersen, ${ }^{a}$ Carol A. G. R. Busk, ${ }^{c}$ \\ Helle Precht, ${ }^{d}$ Boudewijn P. F. Lelieveldt, ${ }^{a, e}$ and Jouke Dijkstra ${ }^{a, *}$ \\ aLeiden University Medical Center, Department of Radiology C2-S, Division of Image Processing, P.O. Box 9600, Leiden 2300 RC, \\ The Netherlands \\ 'Leiden University Medical Center, Department of Medical Statistics and Bioinformatics, P.O. Box 9600, Leiden 2300 RC, The Netherlands \\ 'University of Southern Denmark, Institute of Forensic Medicine, Odense C 5000, Denmark \\ ¿University College Lillebaelt, Conrad Research Center, Odense SØ 5220, Denmark \\ eDelft University of Technology, Intelligent Systems Department, P.O. Box 5031, Delft 2600 GA, The Netherlands
}

\begin{abstract}
Intravascular optical coherence tomography (IVOCT) is an imaging technique that is used to analyze the underlying cause of cardiovascular disease. Because a catheter is used during imaging, the intensities can be affected by the catheter position. This work aims to analyze the effect of the catheter position on IVOCT image intensities and to propose a compensation method to minimize this effect in order to improve the visualization and the automatic analysis of IVOCT images. The effect of catheter position is modeled with respect to the distance between the catheter and the arterial wall (distance-dependent factor) and the incident angle onto the arterial wall (angle-dependent factor). A light transmission model incorporating both factors is introduced. On the basis of this model, the interaction effect of both factors is estimated with a hierarchical multivariant linear regression model. Statistical analysis shows that IVOCT intensities are significantly affected by both factors with $p<0.001$, as either aspect increases the intensity decreases. This effect differs for different pullbacks. The regression results were used to compensate for this effect. Experiments show that the proposed compensation method can improve the performance of the automatic bioresorbable vascular scaffold strut detection. () The Authors. Published by SPIE under a Creative Commons Attribution 3.0 Unported License. Distribution or reproduction of this work in whole or in part requires full attribution of the original publication, including its DOI. [DOI: 10.1117/1.JBO.21.12.126005]
\end{abstract}

Keywords: intravascular optical coherence tomography; intracoronary optical coherence tomography; backscattered intensity; angledependency of near-infrared light; distance-dependency of near-infrared; hierarchical linear regression.

Paper 160559PR received Aug. 22, 2016; accepted for publication Nov. 9, 2016; published online Dec. 7, 2016.

\section{Introduction}

Cardiovascular disease (CVD) is a major cause of death worldwide. ${ }^{1}$ One of the underlying processes that cause CVD is atherosclerosis, which is the long-term accumulation of plaque in the vessel wall. The extent and composition of atherosclerosis can be visualized in vivo with intravascular optical coherence tomography (IVOCT) at a higher resolution of 10 to $20 \mu \mathrm{m}^{2-4}$ compared to other in-vivo imaging modalities, such as intravascular ultrasound (IVUS), computed tomography angiography or magnetic resonance imaging.

IVOCT is an optical imaging modality using near-infrared (NIR) light as the imaging source. The images are acquired using a catheter, which is inserted into the coronary artery. Images of arterial cross-sections are reconstructed from the echo time delay and the intensity of backscattered light. Due to the high scattering of NIR light in blood caused by red blood cells, the artery is flushed with saline or a contrast medium to clear the blood inside the artery. The image intensity is assumed to be only tissue dependent, thus different types of tissue appear different. ${ }^{2}$

In practice, however, the signal magnitude may not be dependent only on the tissue type but also on the position of the catheter with respect to the vessel wall, which causes

*Address all correspondence to: Jouke Dijkstra, E-mail: j.dijkstra@lumc.nl nontissue-related effects on the IVOCT image intensities. ${ }^{5,6}$ An example is given in Fig. 1. The average intensities are calculated within the thin superficial uniform tissue layer of a nonpathological artery segment. Nevertheless, there is a clear variation in the profile of the average intensities [Fig. 1(b)].

The importance of analyzing the effect on intensities caused by the position of the catheter has been well depicted in the field of IVUS. Courtney et al. ${ }^{7}$ showed that the IVUS image intensities are significantly related to the catheter position. Their study concluded that when the distance or the angle toward the luminal wall increases, the intensity will decrease for both intimamedia tissue and adventitia tissue. Earlier work ${ }^{8-10}$ shows that the reflected ultrasound signal is critically dependent on the angle of incidence and varies for different types of arterial plaques.

In the literature of IVOCT image analysis, statistical values of the intensities are commonly used as key features for both automated detection algorithms and the quantitative studies. For example, mean intensity has been applied as one of the textural features for automated tissue characterization. ${ }^{11}$ Recently, a stent strut detection algorithm has been proposed by training a supervised artificial neural network classifier with statistical features including the maximum, mean, median intensities, and so on. ${ }^{12}$ Furthermore, percentile values of intensity distributions are often used as thresholds. For example, the fifth percentile has been used as a threshold for noise removal. ${ }^{13-16}$ Other percentiles were used as cutoff values to determine the trailing 


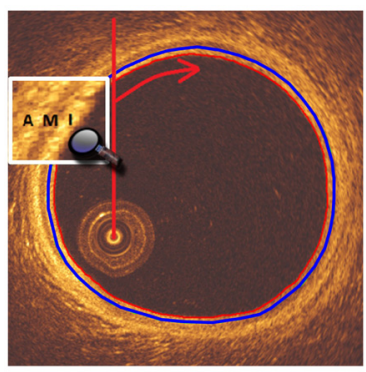

(a)

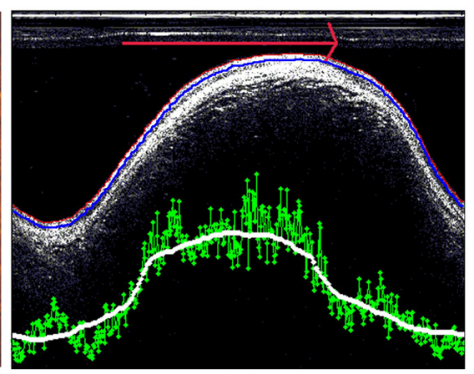

(b)
Fig. 1 (a): An IVOCT image of a nonpathological artery wall: the artery has a regular and almost circular shape; three arterial layers, intima (I), media (M), and adventitia (A), are clearly visible (as shown in zoomed-in top-left corner). (b): Polar representation of the image in (a) sampled clockwise along radial A-lines from the catheter center shown as a bright line on the top of the image. For each A-scan, the average intensity within a superficial thin layer $(\approx 50 \mu \mathrm{m})$ is calculated and shown as the green profile. The white curve is the smoothed green profile.

shadows ${ }^{15,17}$ for metal strut detection, or the black core regions ${ }^{18}$ in the detection of the bioresorbable vascular scaffold (BVS) strut. With the assistance of the BVS strut detection, median and peak intensity values within the black core regions were quantitatively analyzed to track the resorption of the BVS struts over time in IVOCT images at $6,12,24$, and 36 month poststenting, respectively. ${ }^{19}$

The distributions of the intensities can appear different due to the catheter position, which may increase the variation of those statistical numbers. To the best of our knowledge, only one study with respect to this issue on OCT image intensities has been reported which was about nonperpendicular incident light causing a significant variance in the measurement of the articular cartilage. ${ }^{6}$ In the followup studies of the bioresorption progress of the BVS strut, the bias in light intensity caused by the eccentric catheter was claimed to be minimized with normalization by manual selecting reference regions. ${ }^{20}$ Results from IVUS cannot be applied directly to IVOCT, due to the differences in physical properties between both modalities.

The aim of this work is to analyze the effect of the catheter position, with regards to both the distance to the vessel wall and the incident angle of light, on IVOCT image intensities. Based on this analysis, a compensation algorithm is proposed to reduce this effect. As an application of compensated images, images with foam cells have been enhanced and compared with histological cross sections. Furthermore, the compensation algorithm is used in combination with an existing BVS detection algorithm.

The general structure of the paper is as follows. In Sec. 2.1, a light transmission model incorporating both distance-dependent and angle-dependent factors is introduced. In Sec. 2.2, a hierarchical multivariant linear regression model is proposed to further investigate the relationship and estimate both factors. The regression result is further used in Sec. 2.3 to propose a method to compensate images. Results are presented in Sec. 3. Furthermore, a BVS struts detection experiment with the compensated images was carried out in Sec. 4. All the experiments and results are discussed in Sec. 5 with limitations and future works given as well. Conclusions are drawn in Sec. 6 .

\section{Materials and Methodology}

Images of nonpathological segments from nine IVOCT pullbacks recorded with a C7XR swept-source OCT system and a C7 Dragonfly Imaging Catheter (St. Jude Medical, Minnesota) were used. The technical details are listed in Tables 1 and 2 .

\subsection{Distance and Incident Angle Extended Light Transmission Model}

A schematic overview of light propagation for IVOCT imaging is shown in Fig. 2. The light emitted from the catheter first travels through the flush medium before reaching the arterial wall with a distance denoted as $x_{t}$. At the interface between the flush medium and the arterial wall, both reflection and refraction occur. $\theta$ represents the incident angle of the light entering the arterial wall. $\Delta x$ represents the light transmitting distance of the refracted light beam inside the arterial tissue. For the convenience of explanation, we introduce $x=x_{t}+\Delta x$.

\subsubsection{Light transmission model}

As the light propagates inside the arterial wall, the intensity of an OCT signal is typically modeled as the first-order scattering function of $x$ and $\Delta x$ as ${ }^{21}$

$I_{\mathrm{b}}(x) \cong \frac{1}{2} I_{\mathrm{in}} \mu_{\mathrm{b}} T(x) \cdot e^{-2 \mu_{\mathrm{t}} \Delta x}$,

where $I_{\text {in }}$ denotes the light intensity upon entering the arterial wall, $I_{\mathrm{b}}(x)$ denotes the backscattered light intensity from the distance $x, \mu_{\mathrm{b}}$ represents the backscattering coefficient, and $\mu_{\mathrm{t}}$

Table 1 Technical details of the IVOCT system.

\begin{tabular}{|c|c|c|c|c|}
\hline \multirow[t]{3}{*}{$\begin{array}{l}\text { Swept laser } \\
\text { source }\end{array}$} & Center wavelength & $1310 \mathrm{~nm}$ & $\begin{array}{l}\text { Wavelength } \\
\text { range }\end{array}$ & $110 \mathrm{~nm}$ \\
\hline & Sweep rate & $50 \mathrm{kHz}$ & Output power & $20 \mathrm{~mW}$ \\
\hline & Coherence length & $12 \mathrm{~mm}$ & & \\
\hline Pullback & Pullback speed & $20 \mathrm{~mm} / \mathrm{s}$ & Pullback length & $54 \mathrm{~mm}$ \\
\hline Frames & Image frames & 271 & Frame rate & $0.2 \mathrm{~mm}$ \\
\hline
\end{tabular}

Table 2 Number of selected frames in each pullback.

\begin{tabular}{lcccccccccc} 
Pullback No. & 1 & 2 & 3 & 4 & 5 & 6 & 7 & 8 & 9 & Total \\
\hline No. of frames & 28 & 17 & 33 & 21 & 5 & 13 & 14 & 29 & 9 & 169 \\
\hline
\end{tabular}

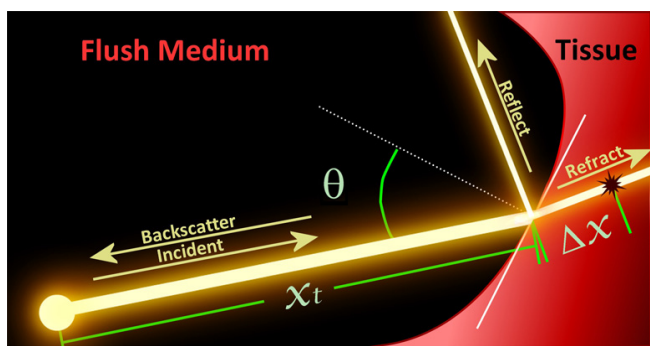

Fig. 2 Light transmission. $x_{\mathrm{t}}$ denotes the distance between the light source and the arterial wall, $\theta$ is the incident angle of the light beam. 
is the total attenuation coefficient (summation of scatter and absorption). $T(x)$ is the confocal function, which is defined as follows: ${ }^{22}$

$T(x)=\left[\left(\frac{x-z_{0}}{z_{\mathrm{R}}}\right)^{2}+1\right]^{-1 / 2}$,

where $z_{0}$ and $z_{\mathrm{R}}$ are the beam waist and the Rayleigh length, respectively.

The intensity entering the luminal wall is affected by two factors: (1) the attenuation in the flush medium region (FMR) and (2) the reflection and refraction at the interface of flush medium and the arterial wall. In a well-flushed artery, the FMR region can be regarded as homogeneous, nonscattering and weakly attenuating, obeying Lambert-Beer law. ${ }^{23}$ With a constant attenuation, $\mu_{f}$, the light decay is determined by the distance from the catheter to the lumen wall, $x_{t}$.

The interaction of the light is more complex at the interface between FMR and the lumen tissue due to the relative irregular surface of the arterial wall. To analyze the effect of the incident angle on image intensities, the total effect of the incident angle is normalized into 0 to 1 by using a term similar to the Fresnel transmission ratio. Thus, $I_{\text {in }}$ is modeled as

$I_{\text {in }} \sim I_{0} \cdot \operatorname{Tr}\left(\theta, n_{i}, n_{t}\right)^{\beta_{2}} \cdot e^{-\mu_{f} x_{t}}$,

where $\beta_{2}$ is the parameter to be estimated. $\operatorname{Tr}\left(\theta, n_{i}, n_{t}\right)$ is the Fresnel like function, which is calculated with the incident angle $\theta$, index of refraction of the incident medium $n_{\mathrm{i}}$ and transmission medium $n_{\mathrm{t}}$, respectively. ${ }^{23}$ With Eq. (3) substituted in Eq. (1) and taking the logarithm from both sides results in

$$
\begin{aligned}
\ln I_{\mathrm{b}}(x) \cong & -\mu_{f} x_{\mathrm{t}}+\beta \ln \operatorname{Tr}\left(\theta, n_{\mathrm{i}}, n_{\mathrm{t}}\right)+\ln T\left(x_{\mathrm{t}}+\Delta x\right) \\
& -2 \mu_{\mathrm{t}} \Delta x+C\left(I_{0}, \mu_{\mathrm{b}}\right)
\end{aligned}
$$

where $C\left(I_{0}, \mu_{\mathrm{b}}\right) \cong \ln \left(I_{0} \cdot \mu_{\mathrm{b}}\right)$ is a constant term.

\subsection{Parameter Estimation of the Linear Model with Hierarchical Linear Regression}

\subsubsection{Hierarchical linear regression}

Hierarchical linear models are specifically utilized for data with hierarchical structures. ${ }^{24}$ Here, a hierarchical linear model is designed to analyze the potential relationship between OCT image intensities and three factors: distance, $(x)$; angle, $\ln \operatorname{Tr}\left(\theta, n_{i}, n_{t}\right)$; and the constant term $C\left(I_{0}, \mu_{\mathrm{b}}\right)$. The linear model for regression is

$$
\ln I_{\mathrm{b}}(x)=\beta_{0}+\beta_{1} \cdot x+\beta_{2} \cdot \ln \operatorname{Tr}\left(\theta, n_{\mathrm{i}}, n_{\mathrm{t}}\right) .
$$

In order to keep the consistency of the notations, the parameters were denoted as $\beta_{0}, \beta_{1}$, and $\beta_{2}$. The A-lines can be hierarchized

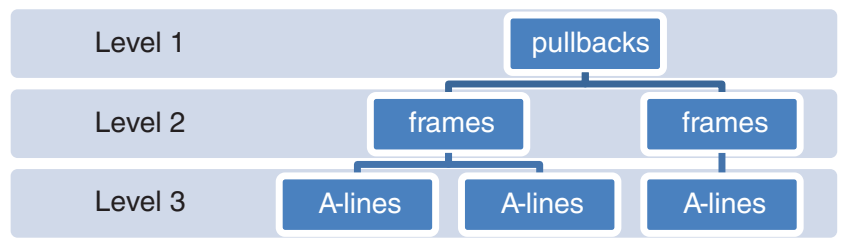

Fig. 3 Multilevel linear model. into different frames, which in turn can be hierarchized into different pullbacks. Based on this observation, a three-level linear model is considered for this study (see Fig. 3).

\subsubsection{Implementation}

The lumen border in the Cartesian images was used to estimate the incident angle. To compensate for the polar to Cartesian transformation, the lumen border points were resampled with respect to the depth. The angle was calculated in a window of nine points.

The index of refraction of the flush solution is 1.449 (read from the stored data). The refraction index of intima is about $1.358 .^{25}$ Therefore, the incident angle is the only variable during the calculation of the transmission ratio for each point.

Intensities of only a thin inner layer of the arterial wall are used for the statistical analysis, then $\Delta x \approx 0$, and thus $x=$ $x_{t}+\Delta x \approx x_{t}$. The general trend of the signal regards to the distance $x_{t}$ is decreasing due to both the attenuation of the flush medium and the confocal function. Approximating this term as linear, the object model for hierarchical linear regression can be written as

$\ln I_{b}(x)=C\left(I_{0}, \mu_{b}\right)+\beta_{1} \cdot x_{t}+\beta_{2} \ln \operatorname{Tr}(\theta)$.

This can be equalized to the hierarchical linear regression model if we denote $\beta_{0}=C\left(I_{0}, \mu_{\mathrm{b}}\right)$; thus, the linear regression can be used to investigate the linear relationship regarding the distance and the incident angle.

\subsection{Compensation}

The linear model that describes the effect of the catheter position can also be used for the compensation of this effect.

Based on the linear regression model, the primary goal for the compensation is to normalize the IVOCT image intensities within the superficial layer of the nonpathological artery. This can be achieved with the following equation involving the regression result:

$I_{\text {compensated }}(y)=\frac{I_{\text {original }}(y) \cdot e^{\hat{\beta}_{0}}}{I_{b}(x)}$.

As defined, $I_{\text {original }}(y)$ and $I_{\text {compensated }}(y)$ denote the original and the compensated IVOCT signals at the depth $y . \beta_{0}$ is the estimated constant term in the regression model. With a thin layer with thickness $\Delta x$ selected, $I_{\mathrm{b}}(x)$ is the average intensity within the superficial thin layer:

$I_{\mathrm{b}}(x)=\int_{y=x_{t}}^{x_{t}+\Delta x} I_{\text {original }}(y) / \Delta x$.

Noting that the following mathematical equation holds

$I_{\mathrm{b}}(x) \sim \hat{\beta}_{1} \cdot \int_{x}^{\infty} I_{\mathrm{b}}(t) \mathrm{d} t$,

where $\hat{\beta}_{1}$ is the estimated parameter. The intensities can be normalized as follows: 


$$
I_{\text {compensated }}(y)=\frac{I_{\text {original }}(y) \cdot e^{\hat{\beta}_{0}}}{\hat{\beta}_{1} \cdot \int_{x}^{\infty} I_{\mathrm{b}}(t) \mathrm{d} t} \cong \frac{I_{\text {original }}(y) \cdot e^{\hat{\beta}_{0}}}{\hat{\beta}_{1} \cdot \sum_{x}^{M} I_{\mathrm{b}}(t)},
$$

where $M$ is a large depth selected far enough away from the lumen border. Noting that

$$
\sum_{y=x}^{M} I_{\text {compensated }}=\frac{e^{\hat{\beta}_{0}}}{\hat{\beta}_{1}}=\text { constant }
$$

the principle of this compensation method is to normalize the summation of intensities behind the lumen border. Since arterial tissues are strong scattering and weak absorbing, the summation of the IVOCT intensities should be approximately constant (linearly related to the total emitting energy from the catheter) for most arterial tissue types; thus, this method is not limited to compensate only the IVOCT images of nonpathological arteries.

\section{Results}

\subsection{Hierarchical Linear Regression}

The hierarchical linear regression considers three fixed effects and two random effects. The $F$-tests result for each of the fixed effects specified in the model indicate that all three effects contribute to the model statistically significantly with $p<0.001$.

Table 3 shows the results of the fixed model. It was found that the constant related to light source $\left(\beta_{0}=6.5121, \mathrm{SE}=\right.$ $0.1615, p<0.001)$, the distance between catheter and artery wall $\left(\beta_{1}=0.0023, \mathrm{SE}=0.0000, p<0.001\right)$ and the logarithm of "Fresnel" transmission ratio $\left(\beta_{2}=2.8178, \mathrm{SE}=0.0725\right.$, $p<0.001)$ were significant predictors.

Table 4 shows the results for the two random factors and residual covariance matrices. Results indicate that both the defined random effects- the frame number $(N u=0.0060$,
$\mathrm{SE}=0.0007, p<0.001)$ and pull-back number $(N u=0.2342$, $\mathrm{SE}=0.1173, p<0.05)$ - contribute to the covariance statistically significantly with almost two thirds of the total variance. However, the influence of the frame number is relatively very small $(\sim 1.6 \%)$ compared to the other contributors. Based on this observation, this random effect can be ignored during modeling.

The histogram of the residual $(\varepsilon=0.1276, \mathrm{SE}=0.0006$, $p<0.0001)$ is distributed symmetrically around zero with a mean value of $-2.01 \mathrm{E}-11$ and a standard deviation of 0.3568 , thus indicating the model can fit the data well.

\subsection{Compensation}

With the results of the linear regression, images can be compensated using Eq. (10) proposed in Sec. 2.3. Figure 4 demonstrates the compensation of IVOCT images of nonpathological arteries. The nonuniform image intensities behind the lumen border and even a small shadow artifact on the lumen wall were compensated. The nonuniform image intensities and even a small unusual shadow on the lumen wall were compensated.

\section{Application of the Compensated Images}

Regarding the application of the compensation algorithm, two experiments were carried out. In the first experiment, compensated IVOCT images with foam cells are compared with the original images assisted with the histological images. In the second experiment, the compensation algorithm was applied to IVOCT images with BVS struts, followed with the strut detection and the evaluation.

\subsection{Foam Cells Visualization}

The compensated images are compared with histological cross sections. Selected frames from two ex-vivo OCT pullbacks on

Table 3 Estimates of fixed effects. ${ }^{a}$

\begin{tabular}{lccccccc}
\hline & & & & & \multicolumn{2}{c}{$95 \%$ confidence interval } \\
\cline { 5 - 8 } Parameter & Estimate & Standard error & $d f$ & $t$ & Sig. & Lower bound & Upper bound \\
\hline$\beta_{0}$ & 6.512 & 0.162 & 8.013 & 40.318 & 0.000 & 6.140 & 6.884 \\
$-\beta_{1}$ & -0.00227 & 0.000 & 79838 & -279.2 & 0.000 & -0.00229 & -0.00226 \\
$\beta_{2}$ & 2.818 & 0.073 & 80041 & 38.848 & 0.000 & 2.676 & 2.960 \\
\hline
\end{tabular}

${ }^{a}$ Dependent variable: natural logarithm of intensities.

\begin{tabular}{|c|c|c|c|c|c|c|c|}
\hline \multirow[b]{2}{*}{ Parameter } & & \multirow[b]{2}{*}{ Estimate } & \multirow[b]{2}{*}{ Standard } & \multirow[b]{2}{*}{ Wald $Z$} & \multirow[b]{2}{*}{ Sig. } & \multicolumn{2}{|c|}{$95 \%$ confidence interval } \\
\hline & & & & & & Lower bound & Upper bound \\
\hline Residual & & 0.128 & 0.001 & 199.864 & 0.000 & 0.126 & 0.129 \\
\hline Intercept (frame) & Variance & 0.006 & 0.001 & 8.541 & 0.000 & 0.005 & 0.008 \\
\hline Intercept (pull-back) & Variance & 0.234 & 0.117 & 1.997 & 0.046 & 0.088 & 0.625 \\
\hline
\end{tabular}

Table 4 Estimates of covariance parameters. ${ }^{a}$

${ }^{a}$ Dependent variable: natural logarithm of intensities. 

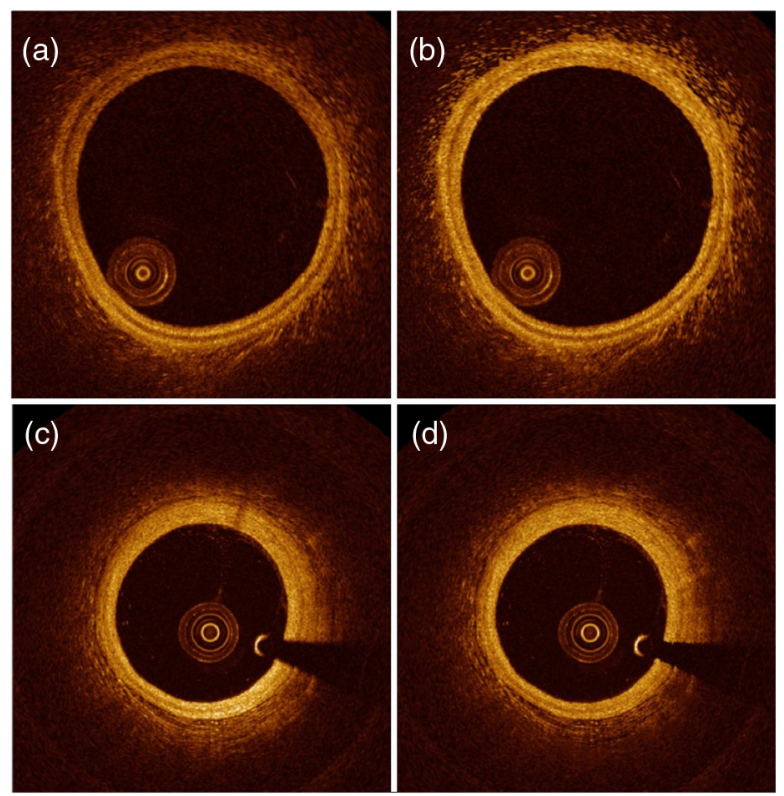

Fig. 4 ( $a$ and $c$ ) The images before compensation and ( $b$ and d) the compensated images. The image in (a) is a clear example of the effects caused by the eccentric position of the catheter, the compensated image (b) shows more evenly distributed intensities around the artery wall. In the second example (c), the light intensities are more or less homogeneous but there is information missing at 1 o'clock. (d) After compensation, the shadow region is removed. explanted hearts ${ }^{26}$ were used. OCT imaging was performed with the Ilumien PCI Optimisation system and a C7 Dragonfly Imaging Catheter of LightLab Imaging, St. Jude Medical, Minnesota. The proximal $5 \mathrm{~cm}$ of the vessels were cut out and standard paraffin embedding was performed. For every $200 \mu \mathrm{m}$, $3-\mu$ m-thick sections were cut and stained with haematoxylineosin. These slices were annotated by a pathologist and matched with the corresponding OCT frames based on anatomical landmarks.

Two examples with bright spots are given in Fig. 5. In Figs 5(a)-5(c), the image intensities marked with red arrows were darkened due to the residual of blood within lumen. In the compensated image, the darkened regions were compensated and the edges between the calcified region and the fibrous region are more clear. In Figs. 5(d) and 5(e), the image regions near side branches were darkened due to the eccentric catheter position. The foam cells, marked with the red arrows, are more accentuated in the compensated image than in the original image.

\subsection{Bioresorbable Vascular Scaffold Strut Detection}

In order to examine the impact of compensating images on automated image segmentation, it is tested on the BVS strut detection, as proposed by Wang et al. ${ }^{18}$ For this purpose, eight pullbacks were used, which were acquired with a C7XR sweptsource OCT system and a C7 Dragonfly Imaging Catheter (St. Jude Medical, Minnesota) at 6 to 12 months poststenting. All the stents are the ABSORB 1.1 BVS (Abbott Vascular, Santa Clara, California). The manual drawn ground truth (GT) data contains 7933 black cores in total. The experiment is carried
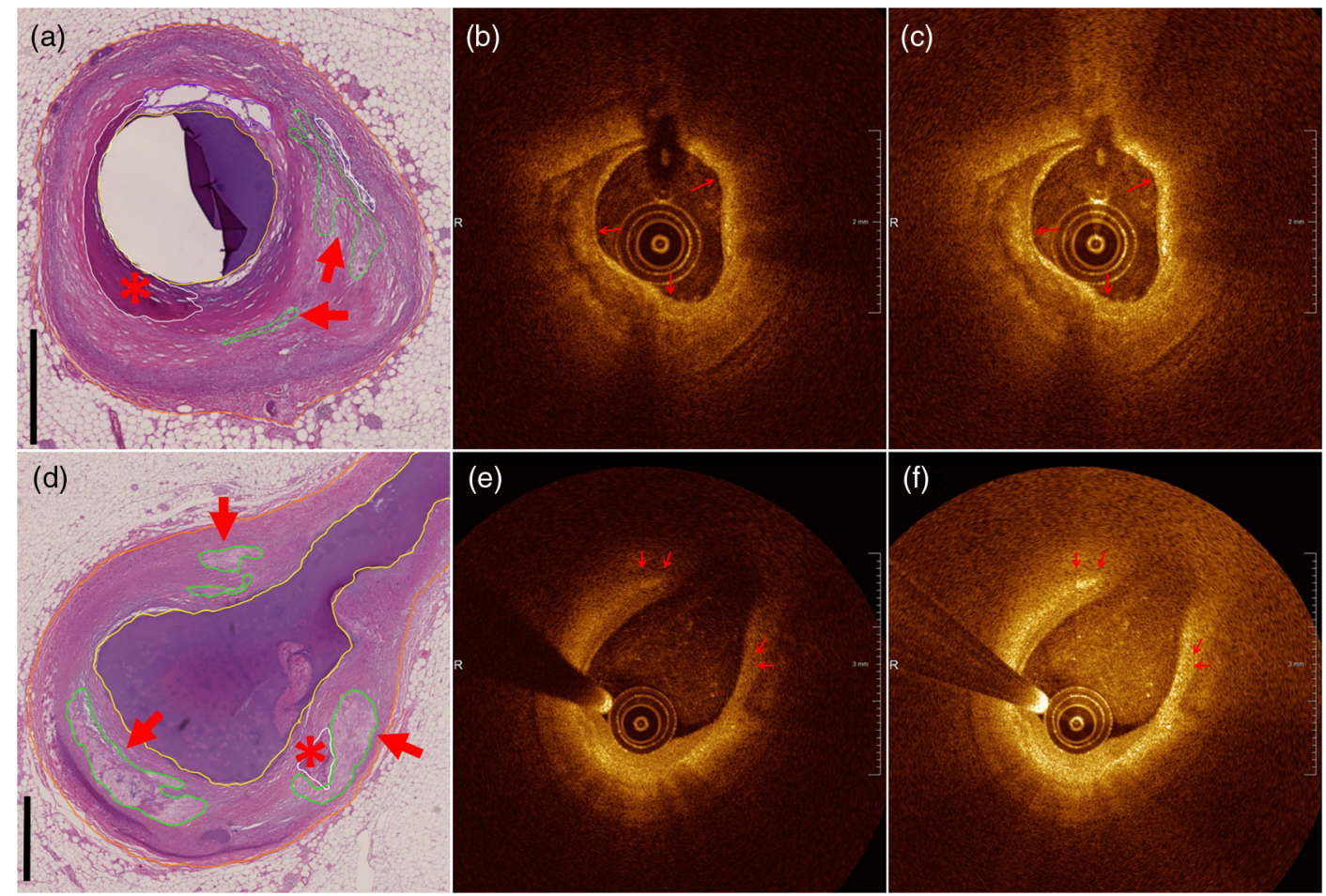

Fig. 5 Demonstration of the effect of compensation. (a-f) From right to left are histology images, original IVOCT images, and compensated images. In the histology images, red arrow indicates the location with form cells, and stars mark the calcified lesions as landmarks. The original and the compensated images are displayed at the same contrast and brightness level, and the red arrows mark noticeable regions for comparison. 
Table 5 The stent struts detection results.

\begin{tabular}{|c|c|c|c|c|c|c|c|c|c|}
\hline \multirow[b]{2}{*}{ Pullback } & \multirow[b]{2}{*}{ No. of GT } & \multicolumn{4}{|c|}{ Original pullback (\%) } & \multicolumn{4}{|c|}{ Compensated pullback (\%) } \\
\hline & & TP & $\mathrm{FP}$ & $\mathrm{FN}$ & $F$-score & TP & $\mathrm{FP}$ & $\mathrm{FN}$ & $F$-score \\
\hline 1 & 776 & 86.5 & 21.4 & 13.5 & 83.2 & 88.9 & 27.3 & 11.1 & 82.2 \\
\hline 2 & 891 & 94.2 & 8.5 & 5.8 & 92.9 & 95.2 & 6.7 & 4.8 & 94.3 \\
\hline 3 & 1158 & 82.6 & 2.7 & 17.4 & 89.1 & 83.9 & 2.3 & 16.1 & 90.1 \\
\hline 4 & 910 & 78.2 & 1.4 & 21.8 & 87.1 & 88.8 & 0.8 & 11.2 & 93.7 \\
\hline 5 & 1389 & 91.9 & 8.9 & 8.1 & 91.5 & 95.2 & 6.9 & 4.8 & 94.2 \\
\hline 6 & 847 & 85.0 & 22.8 & 15.0 & 81.8 & 89.7 & 19.4 & 10.3 & 85.8 \\
\hline 7 & 1059 & 81.7 & 17.2 & 3.6 & 88.7 & 80.6 & 9.3 & 4.6 & 92.1 \\
\hline 8 & 903 & 96.6 & 4.3 & 3.4 & 96.1 & 96.1 & 4.1 & 3.9 & 96.0 \\
\hline Total & 7933 & 87.2 & 10.4 & 10.9 & 89.1 & 89.8 & 8.8 & 8.3 & 91.6 \\
\hline
\end{tabular}

Note: $F$-score $=2 \mathrm{TP} /[2 \mathrm{TP}+(\mathrm{FN}+\mathrm{FP})]$.

out with the in-house developed software QCU-CMS (LUMC, Leiden, The Netherlands).

The compensated pullbacks were rescaled linearly by aligning the 99.5 percentile value of the histogram to that of the original pullbacks, thus the same detection scheme can be applied, as it is described in the work of Wang et al. ${ }^{18}$ The results were evaluated by counting the true positive (TP), the false positive (FP), and false negative (FN), and the $F$-score was calculated as the measurement of the detecting performance. ${ }^{27}$
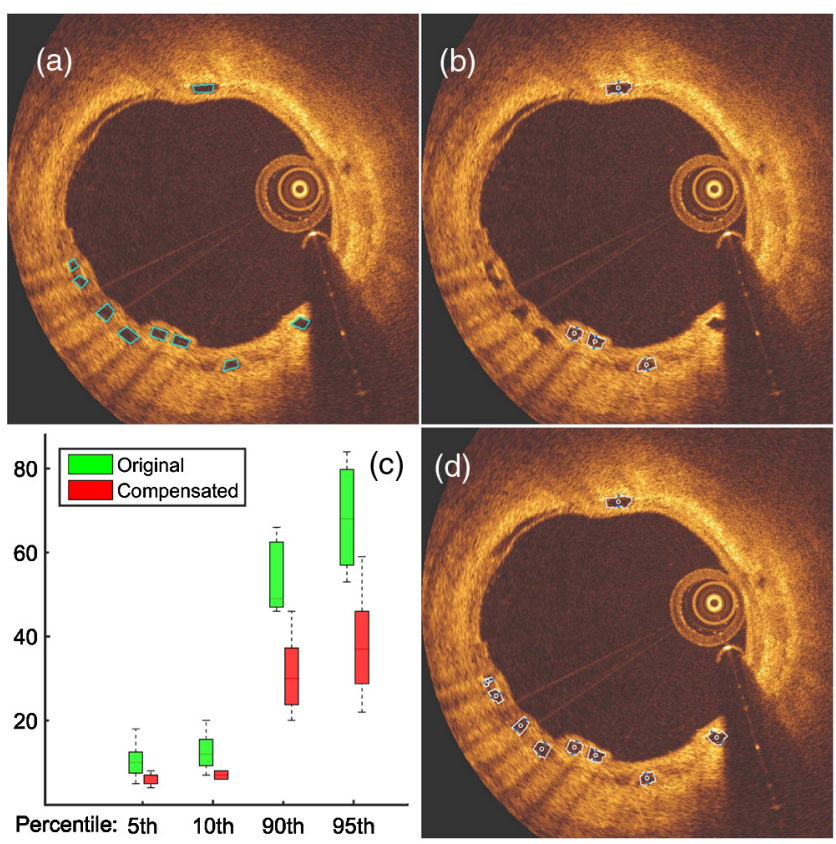

Fig. 6 An example of image with improved BVS detection. (a) The cross section with GT BVS delineated with cyan color. (b) Detection results with the original image delineated with white color. (d) Detection results with the compensated image delineated with white color. (c) Within each black core of the GT, the percentile of both original and compensated image can be seen.
With the described data and experimental settings, the compensated images were used for automatic BVS strut detection. The detection results can be seen in Table 5, where the outperformed F-score is marked with bold font. The compensation algorithm improves the BVS struts detection for 6 out of 8 pullbacks by 1.0 to 6.6 percent in F-score. An example of an improved BVS detection image can be seen in Fig. 6. In QCU-CMS, all the delineations are displayed in the original image. In order to make a clear distinction, the delineations of the GT, the detection results with the original images, and the detection results with the compensated images are shown separated in A, B, and D. The box-and-whisker plot in C shows the intensity percentile within 10 BVS black core regions.

\section{Discussions}

The aim of this work is to investigate the influence of the position of the catheter on IVOCT intensities and to use this knowledge to compensate for it. Two aspects of the catheter's position were analyzed: (1) the distance between the catheter and the arterial wall and (2) the angle of incidence of the light entering the arterial wall.

\subsection{Hierarchical Linear Regression}

The statistic analysis focuses on intima-media regions on the artery wall, thus the tissue-dependent effects were minimized in the study. Similar region selection criteria have been used by Courtney on IVUS images. ${ }^{7}$ The statistical results show that the amount of light that enters the artery wall is significantly related to the catheter position. In the linear model, an anglerelated transmission ratio has been used to model the trend. The trend of this transmission ratio is conforming to empirical observations.

As the angle of incidence increases, the IVOCT intensities decrease accordingly. The more the angle of incidence approaches to a critical angle, the faster the IVOCT intensities decrease. When the angle of incidence approaches this critical angle, light propagation into the tissue in the artery wall is limited. This can explain the "signal dropout" reported by van Soest 
et al. ${ }^{5}$ When the incident angle becomes equal or even larger than a critical angle, there is hardly light entering the artery wall, and the transmission ratio will approximate to zero. This results in the appearance of disconnecting tissue along the arterial wall, which has been reported as dissection artifacts in an IVUS study by Mario et al. ${ }^{10}$ Due to the lack of valid signal, these artifacts cannot be compensated.

As random variables, the pullback number and frame number contribute to the hierarchical linear model significantly in terms of covariance. The covariance contribution of frame number is relatively small enough to be ignored. The covariance of the pullback number occupies almost two thirds of the total covariance. Since the same flush medium was used, this suggests that the distance-dependency can differ between IVOCT imaging catheters. This confirms the statement of van Soest et al. that the parameters of catheters differ from each other. ${ }^{22}$

The range of the estimated incident angles in the experiment is relatively small due to the elliptical shape of the artery wall, which is an inevitable issue of in-vivo IVOCT data. Since the angle related term is between 0 and 1 , the logarithm operation can enlarge the range of the transmission term in the linear model. Another potential issue related to the angle estimation is that the angle of incidence has been estimated with 2-D IVOCT images: the best estimation that can be achieved at present. The estimation can approximate the spatial angle well because the imaging catheter has elasticity to resist over-bending and can thus be assumed parallel to the longitudinal direction of the artery. Nevertheless, it would be interesting to measure the angle of incidence in 3-D in a future study.

\subsection{Compensation}

The compensation of the intensities in the OCT images can enhance the visualization of arterial tissues in IVOCT images. An eccentric position of the catheter can result in inhomogeneous intensity in homogeneous tissue, which requires the contrast and brightness levels to be constantly adjusted during visual inspection. Our proposed compensation algorithm improves the visualization by balancing the signal levels within each pullback, which can be seen in both in-vivo (Fig. 4) and ex-vivo images (Fig. 5).

The principle of the algorithm is that for each pixel at the lumen boundary, the same amount of light enters the tissue, such that further analysis can be carried out without the bias caused by the catheter position. It is designed to overall enhance the absolute intensities for each A-line rather than changing the relative trend. Therefore, it will not affect the parameters like attenuation and backscatter. This is why the proposed algorithm preserves the dark trend within regions with weak backscattering (calcified lesion, dark square inside BVS stent struts, etc) or behind tissue with high attenuation (foam cells, e.g.).

Noting Figs. 5(b)-5(d), shadow artifacts caused by the residual blood in the protective sheath were compensated as well. This is because our compensation algorithm compensates the total energy behind the lumen border for each A-line. The shadow artifacts caused by factors within the lumen, e.g., residual blood and thrombosis, may be the result of a sudden drop of the total energy compared to A-lines in the neighborhood. Therefore, normalizing the total energy can compensate such local shadow artifacts as well.

In the BVS strut detection experiment, the overall result shows that the compensation algorithm can improve the performance of the BVS struts detection. As it is shown in Fig. 6(c), the box-and-whisker plot of the percentile values within the black cores, we can observe that the percentiles in the compensated image are lower than those in the original images. Furthermore, it is worth noting that the lower percentiles over all the black cores are more condensly distributed. This can be the main reason that the proposed algorithm improves detection, since the lower percentiles are used as thresholds in the BVS strut detection. $^{18}$

Meanwhile, there are two pullbacks with lower $F$-score, pullback 1 and 8 . The first pullback was from a patient with a previously implanted metal stent strut followed by a BVS stent treatment, which results in multiple artificial layer structures. This also explains the large amount of the FP in the detection results on the original images in Table 5. The FP ratio on the compensated images is even higher, and this may be because the deeper layer structures are enhanced. Despite a higher FP ratio, the detection with the compensated images gives a higher TP rate. In the eighth pullback, the $F$-score with the compensated image is slightly lower than that with the original images, but it should be noted that both $F$-scores are already very high.

\subsection{Limitations}

There are two catheter-related terms for modeling the IVOCT signal. One is the confocal function and the other is the spectral coherence term. Both terms can be different for different catheters. This can be the reason for the variation that lies in the estimated intercept for the hierarchical regression results. As a result, the compensation factor can be different as well. Noting that the compensation factor serves mainly to normalize the total energy of the incident light onto the arterial tissue, this difference causes only a shift of the histogram of the compensated pullback rather than the shape of the histogram. In these situations, it would be sufficient to apply the compensation algorithm with a fixed "average" factor to remove the bias caused by the catheter position. For the quantification analysis on the data from different pullbacks, ${ }^{19,20}$ the compensating images with fixed factor can be still useful to remove the bias caused by the catheter position, but for further comparison of the statistical numbers, the differences caused by different catheter parameters should be taken into account using statistical tools, such as hierarchical models and so on. It can be useful to characterize the variance caused by different catheters and model it into the compensation method. However, in practice, this will be difficult since most hospitals will not do additional calibration measurements of the catheter before or after the procedure.

Another limitation regarding the catheter-related parameters is that the overall distance-dependent decreasing trend is implemented as a linear regression model after taking the logarithm. So, the algorithm is designed to compensate the general descending trend rather than the Gaussian-shape variation in the confocal function. Taking the numbers reported in the work of Ughi et al. ${ }^{11}$ as an example, $z_{0} \approx 1.5 \mathrm{~mm}$ (focal point) and $z_{\mathrm{R}} \approx 2 \mathrm{~mm}$ (Rayleigh length), the confocal function $T(x)$ at depth $x=4 \mathrm{~mm}$ equals to 0.625 . After the overall linear decreasing trend is compensated, the variation of this term should be around 1 with a variation of $\pm 18 \%$. That is to say, the total incident light is ether overestimated or underestimated by about $18 \%$. For a full depth of $2 \mathrm{~mm}$, the variation of intensities of each pixel can be $0.045 \%$, which is relatively small. 


\subsection{Future Directions}

In this paper, the compensation algorithm shows its potential to be a preprocessing step for automatic BVS stent strut detection. For further incorporating the algorithm into the standard BVS detection workflow, more validation studies are needed, thus the parameters can be further optimized with the compensated images. Meanwhile, it is interesting to see if the compensation algorithm can improve the automatic detection of metal stent struts ${ }^{15}$ as well. Further experiments will be carried out for the metal stent strut detection in the future.

\section{Conclusions}

Both aspects regarding the catheter position, the distance from the catheter to the artery wall and the angle of light incident upon the artery wall, significantly affect IVOCT image intensities. The hierarchical linear regression result shows that as either aspect increases the intensity decreases. Using the hierarchical linear regression result, a compensation algorithm is proposed to enhance the IVOCT images and improve the BVS strut detection. Using the pathological images as a gold standard, it is illustrated that the foam cells appear more accentuated in the compensated image than in the original image. Quantitative evaluation results of the BVS strut detection with the compensated images show that the proposed method improves the performance for the BVS struts detection.

\section{Disclosures}

There are no competing interests to disclose.

\section{Acknowledgments}

Support to Shengnan Liu provided by the Chinese Scholarship Council is gratefully acknowledged.

\section{References}

1. D. Mozaffarian et al., "Heart disease and stroke statistics-2016 update," Circulation 133(15), e599 (2016).

2. G. J. Tearney et al., "Consensus standards for acquisition, measurement, and reporting of intravascular optical coherence tomography studies: a report from the international working group for intravascular optical coherence tomography standardization and validation," J. Am. Coll. Cardiol. 59, 1058-1072 (2012).

3. F. Prati et al., "OCT-based diagnosis and management of STEMI associated with intact fibrous cap," JACC Cardiovasc. Imaging 6, 283-287 (2013).

4. R. Evelyn, A. van Leeuwen, and P. W. Serruys, Eds., Optical Coherence Tomography in Cardiovascular Research, 1st ed., Informa Healthcare, Oxon (2007).

5. G. van Soest et al., "Pitfalls in plaque characterization by OCT: imageartifacts in native coronary arteries," JACC Cardiovasc. Imaging 4(7), 810-813 (2011).

6. Y.-P. Huang et al., "Effects of optical beam angle on quantitative optical coherence tomography (OCT) in normal and surface degenerated bovine articular cartilage," Phys. Med. Biol. 56(2), 491-509 (2011).

7. B. K. Courtney et al., "Effects of transducer position on backscattered intensity in coronary arteries," Ultrasound Med. Biol. 28(1), 81-91 (2002).

8. E. Picano et al., "Angle dependence of ultrasonic backscatter in arterial tissues: a study in vitro," Circulation 72(3), 572-576 (1985).

9. M. de Kroon et al., "Angle-dependent backscatter from the arterial wall," Ultrasound Med. Biol. 17(2), 121-126 (1991).

10. C. D. Mario et al., "The angle of incidence of the ultrasonic beam: a critical factor for the image quality in intravascular ultrasonography," Am. Heart J. 125(2), 442-448 (1993).
11. G. J. Ughi et al., "Automated tissue characterization of in vivo atherosclerotic plaques by intravascular optical coherence tomography images," Biomed. Opt. Express 4, 1014-1030 (2013).

12. H. S. Nam et al., "Automated detection of vessel lumen and stent struts in intravascular optical coherence tomography to evaluate stent apposition and neointimal coverage," Med. Phys. 43, 1662-1675 (2016).

13. P.-Y. Menguy et al., "Preliminary results for the supervised detection of lumen and stent from OCT pullbacks," IRBM 37(5-6), 271-275 (2016).

14. G. J. Ughi et al., "Automatic segmentation of in-vivo intra-coronary optical coherence tomography images to assess stent strut apposition and coverage," Int. J. Cardiovasc. Imaging 28, 229-241 (2012).

15. A. Wang et al., "Automatic stent strut detection in intravascular optical coherence tomographic pullback runs," Int. J. Cardiovasc. Imaging $\mathbf{2 9}$, 29-38 (2013).

16. A. Wang and X. Tang, "Automatic segmentation of vessel lumen in intravascular optical coherence tomography images," in IEEE Int. Conf. on Mechatronics and Automation, pp. 948-953 (2016).

17. F. Dubuisson et al., "Automated peroperative assessment of stents apposition from OCT pullbacks," Comput. Biol. Med. 59, 98-105 (2015).

18. A. Wang et al., "Automatic detection of bioresorbable vascular scaffold struts in intravascular optical coherence tomography pullback runs," Biomed. Opt. Express 5, 3589-3602 (2014).

19. S. Nakatani et al., "Temporal evolution of strut light intensity after implantation of bioresorbable polymeric intracoronary scaffolds in the absorb cohort b trial," Circ. J. 78(8), 1873-1881 (2014).

20. S. Nakatani et al., "Bioresorption and vessel wall integration of a fully bioresorbable polymeric everolimus-eluting scaffold: optical coherence tomography, intravascular ultrasound, and histological study in a porcine model with 4-year follow-up," JACC: Cardiovasc. Interventions 9, 838-851 (2016).

21. T. Valery, Tissue Optics: Light Scattering Methods and Instruments for Medical Diagnosis, 2nd ed., Vol. 23, SPIE, Bellingham, Washington (2007).

22. G. van Soest et al., "Atherosclerotic tissue characterization in vivo by optical coherence tomography attenuation imaging," J. Biomed. Opt. 15(1), 011105 (2010).

23. F. L. Pedrotti and L. S. Pedrotti, Introduction to Optics, 2nd ed., Prentice-Hall International, Englewood Cliffs (1993).

24. L. M. Sullivan, K. A. Dukes, and E. Losina, "Tutorial in biostatistics. an introduction to hierarchical linear modelling," Stat. Med. 18, 855-888 (1999).

25. F. J. van der Meer, "Vascular applications of quantitative optical coherence tomography," Dissertation, Universiteit van Amsterdam (2005).

26. H. Precht et al., "Optimisation of post mortem cardiac computed tomography compared to optical coherence tomography and histopathology," J. Forensic Radiol. Imaging 2(2), 85-90 (2014).

27. C. Goutte and E. Gaussier, "A probabilistic interpretation of precision, recall and f-score, with implication for evaluation," in European Conf. on Information Retrieval, pp. 345-359, Springer, Berlin Heidelberg (2005).

Shengnan Liu is a $\mathrm{PhD}$ candidate at Leiden University Medical Center. She received her BSc degrees in mathematics and applied mathematics in 2009 and MSc degrees in controlling science and engineering in 2012. Her current research interests include intensity analysis, optical properties, and tissue characterization with optical coherence tomography images.

Jeroen Eggermont is a scientific researcher in the Division of Image Processing. He received his MSc degree in computer science from Leiden University in 1998. In 2005, he received his PhD from Leiden University. Currently, his work is mainly focused on the automatic analysis of intravascular ultrasound and optical coherence tomography images.

Ron Wolterbeek is a senior researcher at Department of Medical Statistic and Bioinformatics, Leiden University. His research of interest lies in medical statistics.

Alexander Broersen is a scientific researcher in the Division of Image Processing. He received his MSc degree in computer science at the University of Twente in 2003. He defended his $\mathrm{PhD}$ at Eindhoven University of Technology. In 2008, he started as a postdoctoral researcher at Leiden University Medical Center. His work 
involves the automatic diagnostic vascular analysis by comparing computed tomography angiography with corresponding intravascular ultrasound and IVOCT datasets.

Carol A. G. R. Busk is an undergraduate student in the Forensic Institute at the University of Southern Denmark since February 2015.

Helle Precht is an associate professor in radiography at the University College Lillebaelt. She is particularly interested in digital radiography, radiation protection and imaging quality optimization. She participates in national and international research at the European level.

Boudewijn P.F. Lelieveldt is heading the Divison of Image Processing (LKEB) at the Department of Radiology, Leiden University Medical
Center, Leiden, The Netherlands. He is also appointed as medical delta professor at the Department of Intelligent Systems, Delft University of Technology, Delft, The Netherlands. He serves as a member of the editorial board of Medical Image Analysis and the International Journal of Cardiovascular Imaging, and is an associate editor of IEEE Transactions on Medical Imaging.

Jouke Dijkstra is an associate professor at LUMC-LKEB, where he is the leader of the vascular and molecular imaging section. He has been working at the Division of Image Processing since 1996 and is responsible for the research in vessel imaging, in particular intravascular ultrasound, optical coherence tomography, and computed tomographic angiography. The research resulted in several scientific publications and analytical software packages, which have been transferred to industry and are used worldwide. 